\title{
FORMER LES ENSEIGNANT-E-S DU SECONDAIRE II À L'UNIVERSITÉ PLUS PARTICULIÈREMENT À FRIBOURG, EN SUISSE
}

\author{
EdMÉE RUNTZ - ChRISTAN \\ Université de Fribourg, Suisse
}

\begin{abstract}
REsumé: Cet article donne à voir le rôle de l'université dans la formation des futur-e-s enseignant-e-s du secondaire à Fribourg, en Suisse, en mettant en évidence son interaction avec les écoles. L'organisation générale des études et leur évaluation y sont décrites. Une attention particulière est portée aux partenaires de cette formation universitaire dans les écoles-les enseignant-e-s formateurs-trices-en soulignant leurs rôles, leurs différentes postures et leur formation. L'importance de former des enseignant-e-s à l'université et par l'université est également mise en évidence par le volet recherche qu'implique toute formation académique. En effet, les études de terrain et leurs résultats contribuent à l'amélioration de la formation même des EF.
\end{abstract}

Mots - CLÉs: Formation des enseignant-e-s du secondaire. Les enseignant-e-s formateurs. Interaction école-université.

\section{INTRODUCTION}

En Suisse, chaque canton est responsable de la formation de ses enseignant-e-s quand bien même cette liberté est encadrée par la Conférence des Directeurs cantonaux de I'Instruction Publique (CDIP) qui assure la compatibilité des formations avec les exigences qu'elle a fixées. En effet, il importe que le diplôme délivré permette aux enseignant-e-s du secondaire d'enseigner dans toute la Suisse et pas uniquement dans le canton dans lequel elles ou ils ont été formé-e-s.

Dans le cas qui nous occupe, nous allons nous concentrer sur la formation des futur-e-s enseignant-e-s du secondaire à Fribourg, sur le rôle de la formation à l'université et sur l'importance du terrain dans cette formation. Précisions que celle décrite ici s'adresse aux personnes souhaitant enseigner dans les écoles du secondaire Il préparant à la maturité fédérale ou au diplôme de culture générale. Elle se compose de deux parties : d'une part d'une formation scientifique - portant principalement sur deux disciplines enseignables, dispensées danslesfacultésuniversitairescorrespondantes-, validée par un Master, et d'autre part d'une partie pédagogique et didactique, dispensée par le Centre d'Enseignement et de Recherche pour la Formation à l'enseignement au secondaire (CERF). Cette dernière partie, qui correspond à une formation post-master, s'étend sur deux semestres à temps complet mais peut aussi être suivie à mi-temps sur quatre 
semestres. Elle représente 60 ECTS répartis entre une formation pratique et un volet théorique s'articulant autour de 3 domaines de formation :

- didactique spécifique,

- sciences de l'éducation

- didactique générale et pratique d'enseignement-apprentissage.

Ce dernier domaine s'effectue principalement dans les établissements scolaires du secondaire II sous la forme d'un stage filé de $4 \mathrm{~h} /$ semaine. II implique également une participation active à la vie de l'école. Mais nous aurons l'occasion de revenir sur le déroulement du stage.

Ce cursus permet aux candidates et aux candidats d'acquérir un Diplôme d'enseignement pour les écoles de maturité (DEEM). Il est organisé de manière à articuler des stages autour des savoirs issus des sciences de l'éducation et des didactiques. II porte en principe sur deux disciplines enseignables comme nous le verrons ci-après en développant les aspects de la formation des enseignants telles que :

- la formation:

- les dispositions générales

- le plan d'étude

- l'organisation générale des études

- la validation

la formation professionnelle et le stage
○ cours
○ stage

- les Enseignants Formateurs (EF)

○ leur rôle

- leur formation

\section{FORMATION : DISPOSITIONS GÉNÉRALES}

Un plan d'études ${ }^{1}$ arrête les dispositions générales qui régissent l'admission, la formation et la certification des étudiant-e-s qui souhaitent enseigner à des élèves âgés de 15 à 20 ans qui termineront leurs études par une maturité fédérale ou un diplôme de culture générale. Pour ce faire, les étudiant-e-s doivent obtenir un diplôme d'enseignement pour les écoles de maturité (DEEM) auprès du CERF.

Pour entrer en formation, les futurs enseignants sont soumis à une procédure d'admission dont l'objectif est de:

- Vérifier que l'étudiant-e qui souhaite devenir enseignant-e possède bien un Master/licence académique ou une équivalence qui soit en conformité avec les disciplines de formation et avec les exigences des règlements.

- Clarifier et confirmer le projet professionnel de l'étudiant-e.

- Informer l'étudiant-e des exigences de la formation.

- Associer l'étudiant-e à un ou plusieurs enseignant-e-s formateur-trice-s (EF), répartition qui s'opère en collaboration avec les chefs d'établissement du secondaire Il du canton. 
- Préciser le contrat de formation à établir entre l'EF et le stagiaire, contrat dont la version définitive est déposée une semaine avant la rentrée des classes au CERF et qui sera validé par une commission d'évaluation qui en vérifie la teneur.

- Prouver un niveau C2 dans la langue d'enseignement, dans le cas où l'étudiant n'aurait pas conduit ses études en français ou si sa langue maternelle est autre.

- Fournir les exigences du dossier de candidature (attestations des facultés quant aux diplômes requis, listes des disciplines de formation, formulaire d'inscription, photo récente, CV, extrait de casier judiciaire).

Notons que les candidats ne sauraient être admis ipso facto car les places de stages sont limitées. Les modalités y relatives sont fixées par une ordonnance du Conseil d'Etat. Seule une soixantaine d'étudiant-e-s sont admis chaque année et un quota est fixé par discipline.

\section{PLAN D'ÉTUDE}

Les compétences que les futur-e-s enseignant-e-s doivent acquérir sont listées dans un référentiel qui fait régulièrement l'objet d'une actualisation. En effet, ce référentiel se complexifie et évolue au fur et à mesure de l'analyse rationnelle de l'action intégrée dans une logique institutionnelle et qui prend appui sur la relation pédagogique, conçue comme une ressource.

Ce référentiel doit permettre à l'étudiant-stagiaire de se situer dans les domaines de compétences suivants :

- Domaine didactique: concevoir et piloter des situations d'enseignement/apprentissage en s'adaptant aux élèves et en différenciant les stratégies d'enseignement.

- Domaine pédagogique : favoriser le développement et l'autonomie des élèves ainsi que la relation pédagogique.

- Domaine social : connaître et utiliser de façon appropriée les ressources et règles de l'école pour favoriser l'intégration sociale des élèves et leur développement personnel.

- Domaine du numérique : prendre en compte les enjeux et les défis en lien avec le développement du numérique.

- Domaine du développement professionnel : être en évolution constante vis-àvis de soi-même et vis-à-vis des autres (collègues, autres intervenants).

- Domaine de recherche : connaître et utiliser de façon appropriée les outils de la recherche en sciences de l'éducation. exemples:

Ces compétences génériques se déclinent en divers items, dont voici quelques

- Domaine didactique :

- Concevoir et piloter des situations d'enseignement/apprentissage :

- Maîtriser les savoirs de ses disciplines.

- Transposer ces savoirs.

- Identifier les obstacles didactiques potentiels.

- Formuler des objectifs d'apprentissages.

- Mettre en cuvre et piloter une situation d'enseignement/apprentissage: 
- Communiquer les consignes de manière claire.

- Gérer les interactions avec les élèves et celles des élèves entre eux.

- Evaluer les apprentissages:

- Concevoir des dispositifs d'évaluations adaptés en variant les modalités et les outils.

- Dresser un bilan des acquis des élèves en lien avec les objectifs formulés.

- Recueillir et traiter des informations pour adapter son enseignement.

- Domaine pédagogique

- Etablir une relation pédagogique de confiance en soignant les moments-clés de celle-ci.

- Guider les élèves par des interventions appropriées lors des activités d'apprentissage :

- Choisir des démarches qui favorisent l'engagement de tous les élèves.

- Observer les élèves pour les connaître et identifier leurs difficultés.

- Domaine social

- Mettre en place une gestion de classe efficace :

- Développer des méthodes de travail de nature à favoriser le respect de la diversité et de l'hétérogénéité de la classe.

- Mettre en place des stratégies pour favoriser un bon comportement des élèves.

- Connaître les sanctions et savoir les appliquer.

- Situer son rôle par rapport à celui d'autres intervenants internes ou externes:

- Identifier ses compétences propres.

- Domaine numérique

- Conduire un projet MITIC :

- Développer des connaissances sur les enjeux de développement d'une littératie numérique.

- Acquérir des compétences de plus en plus fines dans le domaine de l'intégration des technologies dans son enseignement.

- Domaine recherche

- Elaborer et analyser des situations d'apprentissage ou de formation:

- Récolter des données dans les branches d'enseignement de l'étudiant-e ou dans le domaine de la formation des EF.

- Analyser et discuter ces données. 


\section{ORGANISATION GÉNÉRALE DES ÉTUDES}

Comme nous l'avons déjà évoqué, le DEEM est une formation duale qui comprend:

1. Une partie scientifique constituée d'un Master, avec parfois des conditions complémentaires qui dépendent de la Faculté et des disciplines concernées. Cette formation doit porter sur deux disciplines enseignables. La possibilité d'une formation avec une seule discipline est acceptée dans certains cas, pour autant que la formation académique dans la branche comprenne au minimum 180 crédits ; cette partie est validée par les facultés idoines qui ont délivré les masters dans les disciplines suivantes:

Faculté des lettres

- Philosophie: Philosophie

- Français: Français, Français: formation de base

- Allemand: Germanistik

- Italien: Italien

- Espagnol: Espagnol

- Anglais: Langues et littérature anglaises

- Russe: Slavistique

- Latin: Philologie classique ou Langue et culture latines

- Grec: Philologie classique ou Langue et culture grecques

- Rhéto-roman: Rhéto-roman

- Histoire: Histoire, Histoire contemporaine, Histoire de la modernité, Histoire générale et suisse de l'époque moderne

- Histoire de l'Art: Histoire de l'Art

- Musique: Musicologie et histoire du théâtre musical

- Enseignement religieux: Science des religions, Sociétés plurielles: cultures, politique et religions

- Pédagogie/Psychologie: Pédagogie/Psychologie

Faculté des sciences

- Mathématiques: Mathématiques

- Informatique: Informatique

- Physique: Physique

- Chimie: Chimie

- Géographie: Géographie

- Biologie: Biologie

- Sport: Sciences du sport et de la motricité

Faculté de théologie

- Enseigement religieux: Théologie, Etude du christianisme et des religions

Faculté de droit / faculté des sciences économiques et sociales

- Economie et droit* : Economie, Droit 
* La branche "Economie et Droit » vaut comme deux branches enseignables : les programmes d'études «Economie » et « Droit » ne peuvent être pris que dans cette combinaison.

Cette formation scientifique étant acquise obligatoirement avant l'entrée en formation professionnelle ne fait pas l'objet d'un développement dans notre article.

2. Une partie pédagogique et didactique comprenant des cours et séminaires universitaires ainsi qu'une formation pratique ; cette partie, gérée par le CERF, correspond à une formation post-master et s'étend généralement sur deux semestres à temps complet. Elle requière une présence des étudiants à l'université et sur le terrain. Elle peut également s'organiser sur quatre semestres à mi-temps, pour autant que l'étudiant-e en fasse une demande motivée. Nous allons particulièrement nous intéresser à décrire les modules de formation et la formation pratique proposée aux étudiant-e-s.

\section{FORMATION PROFESSIONNELLE ET STAGE}

Comme annoncée, cette partie est du ressort du CERF qui I'organise, la dispense et la valide -même si certains cours sont prestés par les sciences de l'éducation. Cette formation théorique est répartie en :

- cours-blocs portant sur les aspects de psychologie de l'adolescent, sur les problèmes de transposition didactique et sur les stratégies d'analyse de séquences d'enseignement;

- organisation des apprentissages et gestion du curriculum;

- retour aux perspectives plus générales ou transversales, en fonction des problèmes rencontrés, lors de la phase de stage en responsabilité.

Rappelons que l'ensemble des modules représente 60 ECTS (Europeen Credit Transfer and accumulation System) qui se répartissent en trois groupes: 


\begin{tabular}{|l|l|}
\hline Matières/Modules & ECTS \\
\hline Didactique spécifique & \\
\hline Didactique Branche 1 & 5 \\
\hline Didactique Branche 2 ou Travail écrit pour DEEM monobranche & 5 \\
\hline Evaluation des apprentissages au secondaire II & 2 \\
\hline Laboratoires didactiques & 4 \\
\hline Culture numérique et intégration des MITIC dans la pratique scolaire & 3 \\
\hline Cours spécifiques & 2 \\
\hline & 21 \\
\hline Sciences de l'éducation et didactique & \\
\hline Didactique générale, pédagogie et gestion de classe & 2 \\
\hline Affectivité en classe & 3 \\
\hline Introduction aux sciences de l'éducation & 1 \\
\hline Classe hétérogène & $\mathbf{1 9}$ \\
\hline Psychopédagogie de l'adolescence & $\mathbf{2 0}$ \\
\hline
\end{tabular}

- Module didactique: orienté vers l'organisation des apprentissages et des enseignements, il vise à concevoir et piloter des situations d'enseignementapprentissage, à donner une vue d'ensemble des outils conceptuels, des procédés et des stratégies d'enseignement-apprentissage. La formation en didactique spécifique se compose ainsi :

- cours et séminaires en synergie avec les questions et difficultés soulevées par la pratique des disciplines enseignées ainsi que celle de leur évaluation

- projet MITIC soutenu par des cours hybrides

- enseignements spécifiques en fonction des besoins des étudiant-e-s.

- Module pédagogique et sciences de l'éducation : Les étudiant-e-s sont amenés à faire des liens entre les théories pédagogiques et l'enseignementapprentissage, à acquérir des connaissances pour enrichir la relation maîtreélève et à développer des habiletés d'analyse de situations complexes. Par l'élaboration d'un portfolio de compétences, les étudiant-e-s entrent dans un processus de réflexivité constante affinant ainsi leur conception éducative, élargissant le champ de leurs possibles pédagogiques. Pour atteindre ses objectifs, le domaine pédagogique propose des cours de : 


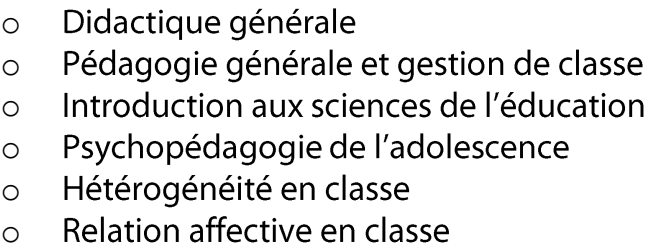

- Module Pratique de l'enseignement-apprentissage: Cette pratique s'effectue dans les établissements scolaires du secondaire ll sous la formed'un stage gradué en quatre phases -observation, collaboration, autonomie et observation activede manière à confier progressivement des responsabilités d'enseignement aux étudiantes et aux étudiants stagiaires.

STAGE

Filé sur un an et gradué, le stage se déroule en 4 périodes par semaine (deux pas discipline enseignée). II concerne l'ensemble des activités éducatives et comprend donc une participation active à la vie de la classe et de l'école. Les stagiaires sont amenés à participer à des sorties culturelles ou sportives, à organiser des journées spéciales, à assister aux réunions de professeurs ou de parents. L'EF veille à ce que cette formation permette aux stagiaires d'apprendre le métier en assurant une répartition équitable de la charge de travail afin de développer petit à petit leur autonomie. Cette formation pratique jouit d'un grand succès auprès des stagiaires qui apprécient de pouvoir confronter les enseignements théoriques acquis à l'université avec les réalités du terrain.

Pendant toute la durée de la formation, les stagiaires participent à l'enseignement d'un ou plusieurs EF dans une ou deux classes, à raison de 148 périodes réparties en quatre phases:

- 1 re phase dite d'observation : 20 périodes

Le stage démarre par un mois d'observation. Ce premier temps de contact doit permettre aux stagiaires de s'intégrer dans l'école, de connaître les élèves, d'étudier le programme d'enseignement. En assistant au cours dispensé par l'EF, les stagiaires notent les démarches didactiques, les prises de décision et la relation entretenue avec les élèves. Ils-elles s'informent des raisons des choix opérés par l'EF qui a alors le souci de fournir toutes les informations et matériel nécessaires aux stagiaires (usage des nouvelles technologies, exigences administratives, plateforme interactive...). Durant cette phase, les stagiaires assistent les EF et collaborent à des tâches limitées (observation et discussion, analyse de la planification, préparation de documents, interventions ponctuelles dans l'enseignement etc.) ;

- 2e phase dite de collaboration: 72 périodes

Les EF et les stagiaires travaillent en partenariat. Les stagiaires prennent progressivement en charge la planification, l'organisation, la réalisation et l'évaluation de séquences didactiques (parties de leçons, leçons entières, 
ensemble de leçons). II arrive aussi que cette phase prenne des formes innovantes : par exemple, le-la stagiaire prépare entièrement une leçon avec tout le matériel nécessaire, mais celle-ci est dispensée par l'EF qui suit à la lettre les propositions de l'étudiant-e ;

- 3e phase dite d'autonomie : 40 périodes

Les étudiant-e-s assument en responsabilité l'ensemble des tâches d'enseignement sous la supervision des EF qui contrôlent les plans de cours, les préparations de séquences mais qui restent la plupart du temps en dehors de la classe durant l'enseignement. Avant chaque séquence ( 6 à 8 leçons), les stagiaires présentent leurs planifications, évaluation comprise, aux EF qui vérifient la faisabilité du travail, les objectifs à atteindre, la variété des stratégies d'enseignement, la corrélation entre enseignement et évaluation, et qui s'en assurent en étant ponctuellement présents en classe (une heure à deux en classe seulement pour l'ensemble de la séquence) ;

- $\quad 4$ e phase : 16 périodes

Les stagiaires reprennent l'observation et participent activement à la vie de l'école en s'impliquant dans des activités sportives ou culturelles avec les élèves ou en les suivant dans leur travail écrit personnel ou leur travail de maturité. Arrivé-e-s en fin de formation, il importe que les stagiaires sortent de l'unique enseignement de leur discipline pour observer et participer aux tâches éducatives de l'école.

\section{VALIDATION}

La validation de la formation est du ressort de la Commission d'évaluation qui dispose des rapports des EF, de ceux des étudiant-e-s, des comptes rendus des visites de stage, des notes obtenues aux évaluations de cours et aux leçons épreuves, pour prendre sa décision. L'ensemble de ces évaluations tend à prouver que les stagiaires ont développé une posture de praticiens réflexifs capables de poursuivre leur développement professionnel tout au long de leur carrière. Elle certifie que les étudiante-s sont en mesure d'enseigner au secondaire II.

\section{EVALUATION DES COURS}

Tous les cours suivis ne sont pas évalués. Seules la didactique des disciplines enseignées, la didactique générale, la pédagogie et gestion de classe, l'introduction aux sciences de l'éducation, les classes hétérogènes, la psychopédagogie de l'adolescent font l'objet d'une évaluation dont la forme est de la responsabilité de l'enseignant qui a proposé le cours. Mais tous les enseignants sont encouragés à faire réfléchir les stagiaires sur leurs connaissances a priori pour les confronter aux connaissances acquises et à leurs mises en pratique dans le cadre scolaire.

\section{EVALUATION DU STAGE}


A deux reprises, à la fin de la phase de collaboration et à la fin de la phase 
d'autonomie, les EF et les stagiaires évaluent le stage en remplissant la grille ci-après qu'ils soumettent à la Commission d'évaluation pour validation.

Nom, prénom du stagiaire
Phase du stage et date

Figure 1 : Grille d'évaluation du stage
DOMAINE OBSERVE
Nom, prénom de l'EF

\begin{tabular}{|c|c|c|c|c|c|}
\hline \multirow[t]{2}{*}{ DOMAINE OBSERVE } & \multicolumn{4}{|c|}{ Appréciations } & \multirow{2}{*}{$\begin{array}{l}\text { REMARQUES } \\
\text { (Veuillez } \\
\text { développer vos } \\
\text { appréciations, en } \\
\text { particulier si elles } \\
\text { se situent dans les } \\
\text { cases "suffisant»" } \\
\text { et «insuffisant»!) }\end{array}$} \\
\hline & $\frac{\frac{c}{0}}{\frac{0}{0}}$ & $\frac{\bar{\omega}}{\mathscr{\omega}}$ & 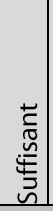 & 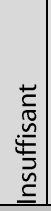 & \\
\hline \multicolumn{6}{|l|}{ 1. Planifier et mettre en acte l'enseignement. } \\
\hline \multicolumn{6}{|l|}{ a. Maîtriser les savoirs disciplinaires requis. } \\
\hline \multicolumn{6}{|l|}{ b. Transposer et transmettre les savoirs disciplinaires requis. } \\
\hline \multicolumn{6}{|l|}{$\begin{array}{l}\text { C. Planifier son enseignement en construisant des situations d'enseignement/apprentissage } \\
\text { adaptées à la réalité de la classe. }\end{array}$} \\
\hline \multicolumn{6}{|l|}{$\begin{array}{l}\text { d. Varier les techniques d'enseignement utilisées pour favoriser au mieux l'apprentissage des } \\
\text { élèves. }\end{array}$} \\
\hline \multicolumn{6}{|l|}{ e. Intégrer dans son enseignement, de façon pertinente et critique, les médias et les TIC. } \\
\hline \multicolumn{6}{|l|}{ f. Concevoir son enseignement en tenant compte de l'ensemble de la formation des élèves. } \\
\hline \multicolumn{6}{|l|}{ q. Citer ses sources et faire preuve d'honnêteté intellectuelle. } \\
\hline \multirow{2}{*}{\multicolumn{6}{|c|}{$\begin{array}{l}\text { h. Autre. } \\
\text { 2. Gérer la classe. }\end{array}$}} \\
\hline & & & & & \\
\hline \multicolumn{6}{|l|}{ a. Etablir des règles explicites (comportement, prise de parole, retard ...). } \\
\hline \multicolumn{6}{|l|}{$\begin{array}{l}\text { b. Ajuster sa posture aux situations rencontrées (présence, voix, espace, gestes, niveau de langage, } \\
\text {...). }\end{array}$} \\
\hline \multicolumn{6}{|l|}{$\begin{array}{l}\text { C. Créer des conditions didactiques et pédagogiques favorables à l'apprentissage et adaptées à la } \\
\text { classe. }\end{array}$} \\
\hline \multicolumn{6}{|l|}{ d. Concilier la gestion du aroupe-classe et les besoins individuels. } \\
\hline \multicolumn{6}{|l|}{$\begin{array}{l}\text { e. Assurer les tâches administratives en conformité avec les règlements (absences, tâches à } \\
\text { domicile,...). }\end{array}$} \\
\hline \multicolumn{6}{|l|}{ f. Gérer les conflits éventuels entre élèves. } \\
\hline \multirow{2}{*}{\multicolumn{6}{|c|}{ a. Autre. }} \\
\hline & & & & & \\
\hline \multicolumn{6}{|l|}{ a. Fixer et communiquer les exiqences attendues. } \\
\hline \multicolumn{6}{|l|}{$\begin{array}{l}\text { b. Prendre en considération et appliquer différentes méthodes d'évaluation des effets de son } \\
\text { enseignement. }\end{array}$} \\
\hline \multicolumn{6}{|l|}{$\begin{array}{l}\text { C. Prendre en compte et traiter des informations en vue d'adapter l'enseignement (évaluation } \\
\text { diagnostique). }\end{array}$} \\
\hline \multicolumn{6}{|l|}{ d. Dresser un bilan des acquisitions faites et encore à faire (évaluation formative et sommative). } \\
\hline \multicolumn{6}{|l|}{ e. Communiquer clairement la situation des/de l'élève-s par rapport aux exiqences. } \\
\hline \multicolumn{6}{|l|}{ f. Proposer des remédiations. } \\
\hline \multirow{2}{*}{\multicolumn{6}{|c|}{ a. Autre. }} \\
\hline & & & & & \\
\hline \multicolumn{6}{|l|}{ a. Favoriser l'intéaration de chaque élève dans le aroupe-classe. } \\
\hline b. Susciter l'intérêt des élèves. & & & & & \\
\hline $\begin{array}{l}\text { C. Prendre en considération les préalables (anticiper les difficultés, connaître les situations socio- } \\
\text { culturelles, affectives, scolaires, ...) aux situations d'apprentissages. }\end{array}$ & & & & & \\
\hline d. Amener les élèves à collaborer entre eux et à assumer des tâches en autonomie. & & & & & \\
\hline $\begin{array}{l}\text { e. Respecter les convictions des élèves compatibles avec le respect du groupe et de chacun de ses } \\
\text { membres. }\end{array}$ & & & & & \\
\hline f. Conserver une bonne distance avec ses élèves. & & & & & \\
\hline a. Sanctionner adéquatement. & & & & & \\
\hline h. Autre. & & & & & \\
\hline
\end{tabular}




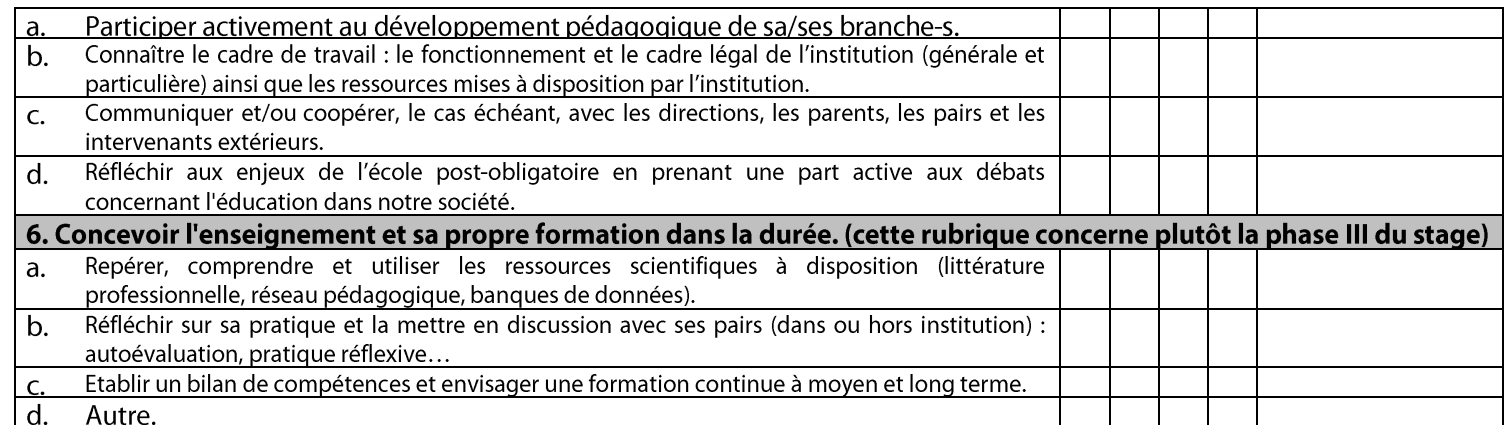

Remarques, commentaires:

A mon avis:

compétences suffisantes pour que la formation soit validée.

besoin de prolonger cette phase.

nécessité de refaire cette phase.

Lieu et date :

Signature

\section{LEÇON ÉPREUVE}

Outre la réussite du stage, les stagiaires doivent encore présenter deux leçons examens, une par discipline d'enseignement. Deux examinateurs ou examinatrices au moins assistent aux leçons d'épreuve (LE): un représentant ou une représentante du domaine d'études de la formation à l'enseignement et le didacticien ou la didacticienne de la branche. En outre, sont invité- e-s à prendre part aux leçons d'épreuve: un représentant ou une représentante de la discipline concernée à I'Université, I'EF et l'enseignant-e de la classe dans laquelle la leçon d'épreuve est présentée.

L'objectif des LE est de vérifier que les stagiaires mettent en pratique les éléments théoriques et didactiques de leur formation, transmettent un contenu scientifique de façon adéquate aux élèves, assurent la gestion de la classe et proposent une analyse pertinente de leur pratique.

Le jury assiste à 45 minutes de LE. Après cette leçon, un entretien a lieu entre le jury et l'étudiante ou l'étudiant durant environ 30 à 45 minutes au maximum. Le jury est présidé par la représentante ou le représentant du CERF et a pour fonction de permettre une évaluation de la leçon et de son analyse. 
La représentante ou le représentant du CERF introduit l'entretien en explicitant la démarche, puis c'est à la candidate ou au candidat de s'exprimer. Les membres du jury peuvent poser des questions de clarification ou d'explicitation. Ils sont ensuite invités à mettre en évidence les points forts et les limites observés quant à la préparation, à la leçon et/ou à son analyse relativement à leur domaine d'expertise, sans pour autant porter un jugement quant à la note ou à l'appréciation globale. Suite à cela, en l'absence de la candidate ou du candidat, le jury délibère sur la note et la fixe à partir des indicateurs suivants: la pertinence du travail préparatoire dans sa globalité et le sérieux de cette préparation ; la mise en œuvre de ce qui a été anticipé et la capacité à s'adapter à la réalité de la classe ; I'analyse réflexive faisant suite à l'enseignement. Dans ce contexte, le statut de l'erreur a un rôle particulier : une erreur est toujours possible, mais ce qui est déterminant pour les membres du jury, c'est la capacité de la stagiaire ou du stagiaire à la percevoir, à l'analyser et à proposer des remédiations.

\section{ENSEIGNANTES FORMATRICEES, ENSEIGNANTS FORMATEURS}

Selon Lamaurelle (2010), il faut accompagner pour former des enseignants efficaces et la qualité de la formation du futur enseignant repose en grande partie sur les EF. En effet, ceux-ci sont des acteurs importants de la formation des enseignants. De ce fait, ils doivent disposer de compétences professionnelles, être expérimentés, et bien formés pour assurer un encadrement efficace. N'importe quel enseignant ne peut pas prétendre être EF sous prétexte qu'il a de l'expérience, la pratique enseignante n'étant pas en soi formatrice pour le stagiaire. Les EF doivent disposer de savoirs formels et expérientiels pour accompagner, c'est à dire « être avec et aller vers» (Paul, 2006)².

\section{RÔLE LES EF}

Mais cette formule suffit-elle aux EF pour connaitre leur rôle dans la formation des jeunes collègues? Comme la pratique d'accompagnement a une grande influence sur les développements des futurs professionnels (Crasborn, Hennissen, Brouwer, Korthagen \& Bergen, 2011), il importe de former les EF pour que l'accompagnement devienne une sorte de partenariat professionnel de deux ou plusieurs personnes dont les rôles sont très bien définis (Gadusova et Svarbova, 2010): l'accompagné-e doit être en mesure de se décentrer et ainsi de s'observer travaillant en analysant son enseignement (Gaté \& Charrier, 2011). L'accompagnateur-trice doit également adopter une attitude réflexive, afin d'« être sensible à la manière dont les stagiaires reçoivent sa demande et ses analyses » (Altet, 2011, p.32). Par ailleurs, l'EF se doit d'être structurant-e, en évitant de juger, afin que l'enseignant-e novice puisse se construire. Le but de l'accompagnement est d'aider le stagiaire à entrer dans la vie professionnelle, de lui permettre de s'émanciper petit à petit en visant à « favoriser le transfert des acquis issus de la formation en situation de travail et le développement de compétences et d'identités professionnelles » (Wittorski, 2006, p.27). Cette émancipation professionnelle est le but visé. Pour y parvenir, en fonction de l'expérience du stagiaire et de la personnalité de l'EF, diverses postures sont possibles ${ }^{3}$ : 


\section{Posture d'imposeur :}

L'imposeur ressemble à l'«imperator» de Crasborn et al. (2011). C'est l'EF qui prend les initiatives lors de l'analyse de pratiques, ne laissant guère la parole au stagiaire, lui donnant des consignes strictes et une marche à suivre. La transmission du métier se fait par imitation car le stagiaire doit faire comme l'EF ou du moins comme ce-dernier le préconise. Les formulations usitées ressemblent à :

- je vais donc commencer par mes observations ;

- donc tu dois toujours bien donner les consignes, clarifier les choses avant de former les groupes et d'attribuer à chacun une tâche ;

- tu dois ..., il faut que tu ... ».

\section{Posture d'organisateur :}

L'organisateur est conscient que le stagiaire a besoin que l'on balise le chemin et décide de fixer des objectifs à atteindre. Le stagiaire n'a pas identifié ses forces et ses faiblesses, il reste centré sur le savoir à transmettre. On est proche de la perspective de l'ouvreur pour Paul (2016). L'EF, dans le cas d'une analyse de pratiques, serait amené à dire:

pour cet exercice, j'aurais apporté une ou deux variantes supplémentaires ;

- $\quad$ tu devrais commencer par les mettre sur l'exercice et ensuite les stopper pour leur demander d'expliquer leur démarche;

- en demandant aux élèves de faire cette recherche quel est ton but ? Reformule ta consigne en fonction de cet objectif.

\section{Posture de co-constructeur :}

Cette posture se situe entre les deux pôles. Elle divise l'autonomie en deux. Le stagiaire et l'EF travaillent, planifient et réfléchissent ensemble. Cette posture permet une discussion ouverte et parfois une réflexion à deux sur un moyen de régulation. À la suite d'un échange, l'EF pourrait utiliser cette formulation :

- $\quad$ ça ne t'empêche pas pour la suite de quand même imprimer et distribuer ce schéma que tu avais préparé pour les élèves;

- $\quad$ essaie d'abord de t'approcher de celui qui bavarde et seulement après tu interviens oralement, comme tu l'as fait, en rappelant le règlement;

- comme alternative, je peux te proposer de faire une mise en commun par rapporteurs successifs.

\section{Posture de facilitateur :}

Cette posture laisse de plus en plus d'autonomie au stagiaire, car le facilitateur reconnaît les compétences du stagiaire et lui laisse la place nécessaire. L'EF interviendra si nécessaire et/ou à la demande du stagiaire. L'EF est considéré comme une personne ressource, il doit donc utiliser des formulations comme:

- $\quad$ si tu as des hésitations tu peux toujours m'écrire ;

- n'hésite pas à me demander si tu as besoin de conseils ;

- $\quad$ tu connais les capsules vidéos sur le sujet ? Elles peuvent te servir d'alternative.

\section{La posture d'émancipateur :}


L'émancipateur laisse une autonomie maximale au stagiaire. II laisse le stagiaire chercher son identité professionnelle en restant bien entendu là en cas de besoin. Le stagiaire vivra une situation qui lui permet d'expérimenter sa future activité professionnelle. Cette phase d'autonomie et de professionnalisation peut se traduire par: tu es libre d'organiser la dernière partie du cours comme tu l'entends;

- pour la suite du cours, je te laisse choisir quels exercices iraient le mieux;

- $\quad$ n'hésite pas à utiliser d'autres sources. Je sais que les collègues ont laissé des choses intéressantes sur la plateforme de l'école.

Mais être dans une posture d'accompagnement ne dispense pas l'EF d'observer, de mener des entretiens d'explicitation et d'évaluer son-sa stagiaire. C'est pourquoi la formation que nous proposons s'oriente vers ces objectifs à atteindre. En effet, la mission des enseignant.e.s formateurs et formatrices (EF) est double :

- accompagner les étudiant.e.s, tout au long de leur formation et en particulier pendant leurs stages

- participer à l'évaluation des compétences des stagiaires.

Ce travail d'accompagnement se déroule notamment lors d'entretiens réalisés à la suite de séances d'observation de séquences d'enseignement.

\section{FORMATION}

Afin d'offrir un soutien à la fonction d'EF, le CERF -en collaboration avec le ZELF (centre de formation des enseignant-e-s alémaniques), la didactique universitaire et la HEP (haute école pédagogique qui forme des enseignante-s primaires)- a mis sur pied un programme de formation. L'objectif de cette formation est de permettre aux EF de choisir ce dont ils ont besoin pour accompagner au mieux leurs stagiaires.

Certains modules favoriseront une approche réflexive, développeront les capacités d'analyse, amélioreront les techniques d'entretien ou d'évaluation ; d'autres permettront de discuter les aspects didactiques de la formation; d'autres aideront à gérer les conflits ou à travailler sur la résonance dans la relation; d'autres encore serviront à transmettre une passion.

\section{COMPOSITION}

Le programme complet de formation comporte 20 demi-jours et peut être suivi sur plusieurs années. La plupart des modules comportent 4 demi-jours de formation. Certains, comme les conférences, ne représentent qu'une demi-journée. D'autres sont proposés en langue allemande afin de donner l'occasion d'approfondir la connaissance de la philosophie de l'enseignement alémanique.

La possibilité d'abandonner un module est également offerte lorsque l'EF juge que le module choisi ne répond pas à ses besoins.

\section{MODALITÉS DE TRAVAIL}


En général, des mises en situations (confrontations de pratiques, d'analyse de séquences vidéo, d'extraits d'entretien de formation) alternent avec des apports théoriques et des exercices de réinvestissement.

La formation attestée équivaut à 5 crédits. Cependant, celle-ci peut être prolongée par un CAS Certificate of advanced studies spécifique à la formation des Enseignant-e.s-Formateurs/trices intéressé-e-s à élargir leurs capacités d'encadrement des stagiaires.

\section{BUTS DU CAS}

Après l'obtention du certificat, les EF certifiés sont amenés à :

- Accompagner et évaluer plus efficacement vos stagiaires.

- Conseiller les enseignant-e-s- nouvellement engagé-e-s.

- Coopérer aux projets d'établissement.

- Initier ou soutenir des recherches en pédagogie.

\section{Composition du CAS}

Le CAS équivaut à 15 ECTS correspondant à environ 450 heures de travail. Après la formation initiale attestée (20 demi-jours), le cursus sera complété pour atteindre 30 demi-jours de cours. Pour le quart restant de la formation, il sera nécessaire de suivre des stagiaires et de rédiger un travail écrit de certification (TEC) de 30 pages en rapport avec la fonction d'EF, sous la direction d'un-e spécialiste proposé-e par le CERF. Les modules proposés prolongent ceux de la formation initiale et s'articulent autour des thématiques suivantes:

\section{Observer}

Pour que l'observation soit un outil de progression, il est nécessaire qu'il y ait discussion entre le stagiaire et l'EF afin que soit évité l'écueil où «l'un soit repéré comme voyeur et l'autre comme modèle à imiter. Chacun doit chercher à identifier son action pédagogique et confronter l'image qu'il se fait de lui à celle qu'un observateur a de lui » ((Leduc-Claire et Py, 2005, p.79). C'est une collaboration orientée vers la modification et la progression des pratiques du stagiaire.

Les différents modules ayant trait à l'observation du stagiaire ont pour objectifs de se situer comme observateur de son stagiaire avant d'en être évaluateur. Le but est d'avoir des outils à disposition afin d'éviter de se tourner vers une interprétation de l'observation et d'échanger sur les critères qui font qu'une leçon sera définie comme bonne.

\section{Savoir mener des entretiens d'explicitation}

Un entretien se veut productif et efficace, et pour ce faire, les modules préparent les EF à mieux conduire les réunions avec leurs stagiaires. Les EF sont sensibilisés aux différents types de réunions existantes, l'objectif étant d'aider le stagiaire à mettre en mots sa pratique réelle afin d'en faire un levier d'apprentissage. 


\section{Evaluer}

Les modules centrés sur l'évaluation proposent des outils pour évaluer le stagiaire, d'une manière formative et certificative, dans le but de concevoir une grille propre à chaque EF et à la discipline enseignée. La sensibilisation aux outils et aux grilles d'évaluation est nécessaire afin que le processus d'évaluation soit juste, de bonne qualité, objectif.

\section{Communiquer avec son stagiaire}

Certains modules sont destinés à favoriser la communication entre les EF et leurs stagiaires: comment par exemple partir d'une situation critique pour la rendre productive. Ces modules fournissent des outils qui sensibilisent aux stratégies et aux formes de dialogues adéquates.

Un large choix de modules est ainsi offert aux EF afin de leur permettre de suivre au mieux leurs stagiaires.

\section{CONCLUSION}

Arrivé au terme de cette description de la formation des enseignant-e-s et des enseigant-e-s fromateurs-trices pour le secondaire II à Fribourg, il nous importe de souligner combien il est précieux que l'université ait en charge cette tâche. En effet, contribuer à la formation des futur-e-s enseignant-e-s permet d'orienter l'éducation des jeunes. En ajoutant la formation des EF à la formation initiale des enseignant-e-s, I'université met un pied dans les établissements scolaires du canton. De cette manière, il lui devient plus aisé de connaître le terrain, de mener des recherches appropriées aux exigences ou aux souhaits du terrain.

Pour répondre aux besoins des différents acteurs, il importe que la formation puisse largement s'inspirer de la recherche et que celle-ci soit réalisée à leur demande. Pour n'en donner qu'un exemple, nous allons montrer ici combien cette interaction est importante. Pour ce faire, nous allons évoquer une recherche en cours dont l'objectif est I'amélioration de la formation des EF par leur stagiaire. En effet, de précédentes recherches ont mis en évidence un décalage important entre les déclarations des EF quant à leur posture et la réalité observée. Si les formateurs-trices désirent idéalement rendre leur stagiaire de plus en plus autonome en passant d'une posture de co-constructeur à une posture d'émancipateur, concrètement il n'en est rien. Au fur et à mesure que le stage évolue, les EF restent fidèles à leur façon de faire, sans grande variation de leur posture initiale. Lorsqu'ils bougent, ils le fond naturellement vers une posture de plus en plus dirigiste. Tout se passe comme si en fin de stage il y avait urgence: dispenser les derniers conseils, donner les stratégies à privilégier pour que les élèves apprennent, transmettre leur expérience. Comment alors aider ces EF à devenir les accompagnateurs qu'ils souhaitent être? 
Nous avons imaginé que cet apprentissage pouvait être soutenu par les stagiaires de la manière suivante: En début de stage, ils soumettent à leurs EF le questionnaire ciaprès sur les postures en leur demandant de répondre rapidement aux items proposés.

\section{Figure 2 : Questionnaire sur les postures ${ }^{4}$}
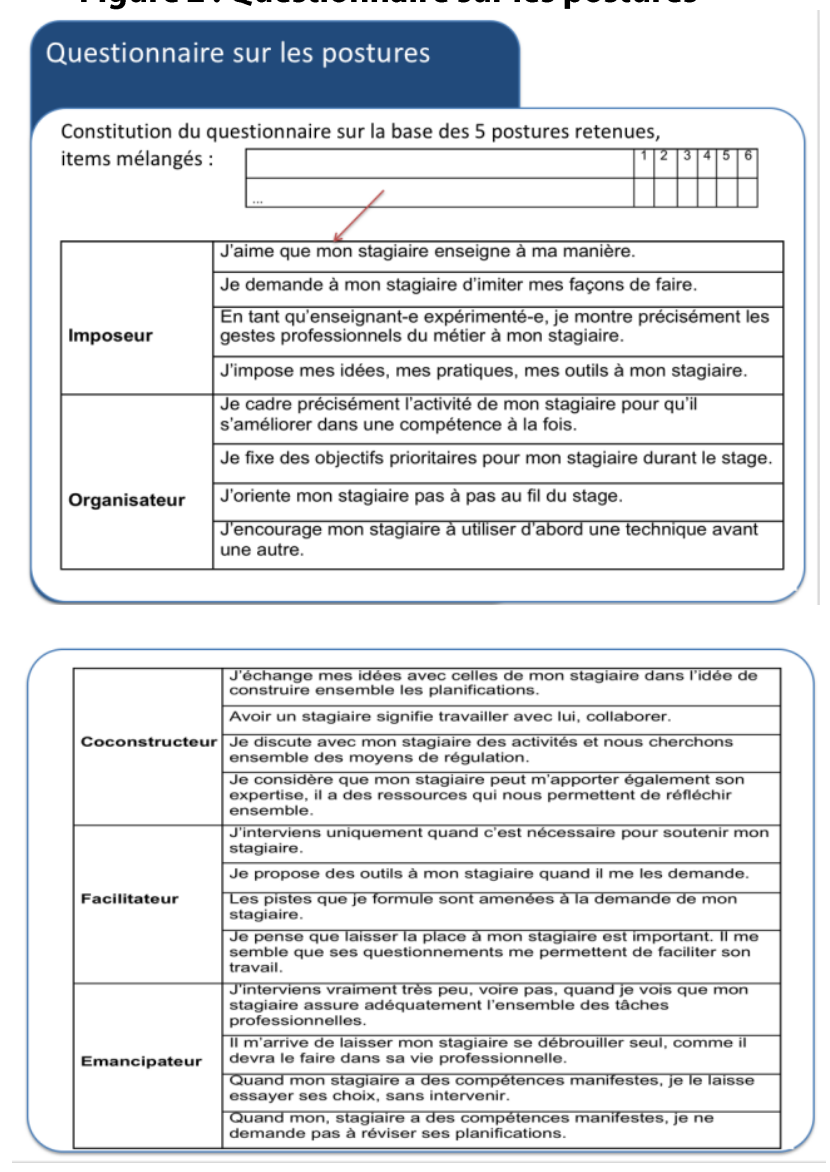

Après un mois de stage, un premier entretien d'explicitation est enregistré et retranscrit. Les verbatims sont ensuite analysés par les stagiaires dans le cadre du laboratoire de didactique. Une méthode inter-juge permet de valider le résultat de cette analyse. Les postures privilégiées par les EF sont mises en évidence et comparées aux postures idéalisées qui étaient ressorties du questionnaire. Ces résultats sont ensuite présentés aux EF, discutés, expliqués, exemplifiés. Un document faisant état des cinq postures exemplifiées est laissé aux EF. C'est alors que le-la stagiaire commence son travail de "formateur-trice». Pour ce faire, le-la future enseignant-e fait un exercice de praticien-ne réflexif-ve, s'interrogeant en présence de son EF sur les raisons qui ont fait qu'une posture a été choisie, plutôt qu'une autre: «C'est parce que je n'ai pas su gérer la classe au moment de la répartition des élèves par groupes que tu me dis de travailler avec des feuilles de couleur et que tu m'expliques comment tu procèdes? Tu as raison, ta manière d'organiser le travail me semble efficace. » Les stagiaires choisissent alors de faire 
ressortir les postures utilisées par leur EF, sans porter de jugement de valeur sur celles-ci, tout en soulignant les prises de paroles empreintes de bienveillance, de confiance, de valorisation. A la fin de la phase II, de nouveaux entretiens seront enregistrés et analysés afin de voir comment les EF se positionnent. Depuis quatre ans nous notons que les EF évoluent peu dans leur posture de formateur-trice et quand ils-elles le font, $c^{\prime}$ est presque toujours dans le sens de diriger les stagiaires, de leurs donner des exemples à suivre. Nous sommes dons curieux de voir si l'information et l'ébauche de formation sur les postures des accompagnateurs-trices a un effet sur les EF. Mais ce qui est sûr, c'est que cette recherche qualitative longitudinale portant sur une quinzaine de situations d'accompagnement -chaque année autour des stagiaires du secondaire II- nous permettra sans doute d'améliorer la formation et l'accompagnement par et pour la recherche universitaire.

Article reçur le: 04/02/2019

Approuvé pour publicarion dans: 27/06/2019

\section{FORMAÇÃO DE PROFESSORES DO SECUNDÁRIO II NA UNIVERSIDADE, MAIS PARTICULARMENTE EM FRIBOURG, SUÍÇA}

RESUMO: Este artigo mostra o papel da universidade na formação de futuros professores do ensino médio em Fribourg, Suíça, destacando sua interação com as escolas. A organização geral dos estudos e sua avaliação são descritas. É dada especial atenção aos parceiros desta formação universitária nas escolas - os professores-formadores - enfatizando seus papéis, suas diferentes posturas e sua formação. A importância da formação de professores na universidade e através da universidade também é destacada pelo componente de pesquisa que envolve toda a formação acadêmica. De fato, estudos de campo e seus resultados contribuem para melhorar o treinamento das próprias RUs.

PALAVRAS - CHAVE: Formação de professores do ensino secundário. Formadores de professores. Interação escola-universidade.

\section{TRAINING SECONDARY TEACHERS AT THE UNIVERSITY MORE PARTICULARLY IN FRIBORG, SWITZERLAND}

ABSTRACT: This article shows the role of the university in the training of future secondary school teachers in Friborg, Switzerland, highlighting its interaction with schools. The general organization of the studies and their evaluation are described. Particular attention is paid to the partners of this university training in schools - the teachers-trainers - emphasizing their roles, their different postures and their training. The importance of training teachers at the university and through the university is also highlighted by the research component that all academic training involves. Indeed, field studies and their results contribute to improving the training of RUs themselves. 
KEYWORDS: Training of secondary school teachers. Teacher-trainers. School-university interaction.

\section{NOTES}

1) Université de Fribourg, CERF, plan d'études pour l'obtention du diplôme d'enseignement pour les écoles de maturité DEEM consulté le 29.12.2018:

https://www3.unifr.ch/cerf/fr/assets/public/pdf/doc/PlanEtudesDEEM.pdf

2) Paul, M. (2006), L'accompagnement: quels enjeux pour le tutorat. Colloque tutorat et Accompagnement, pp 11-24, Bordeaux : AIFRISSS.

3) Modèle proposé par le TREFL : travail du groupe de recherche des universités de Trois Rivières, Fribourg et Louvain sur les postures et modalités des accompagnateurs de stagiaires.

4) Cette grille, mise en forme pour l'analyse des entretiens d'explicitation entre le formateur et son stagiaire sera présentée dans la revue Phronésis sous le titre «Emergence d'une grille d'analyse de l'accompagnement des stagiaires pour améliorer la formation à l'enseignement 》 (Séphane Colognesi, Christine Lebel, Edmée Runtz-Christan, Catherine Van Nieuwenhoven, Louise Bélair).

\section{BIBLIOGRAPHIE}

ALTET, M. (2011). La construction de la professionnalité des enseignants débutants : la place des maîtres-formateurs dans le cadre d'un héritage des IUFM remis en question. In J.-Y. Robin \& I. Vinatier (Eds.), Conseiller et accompagner. Un défi pour la formation des enseignants, (pp. 19-37). Paris: L'Harmattan.

CHARLIER E., BIÉMAR S. (2012). Accompagner : un agir professionnel, préface : Anne Jorro. De Boeck, Bruxelles.

GADUSOVA, Z \& SVARBOVA, E. (2010). Formation des enseignants au mentorat. In J.-C. Loos, A. Malderez, M. L'Hostie, R. Gauthier, A. Henrion, C. Levêque et all. (Eds.), L'accompagnement des nouveaux enseignants. Mentoring Newly-Qualified Teachers, ( $p$. 161-174). Bruxelles : Editions Modulaires Européennes \& InterCommunication sprl.

GATE, J.-P. \& CHARRIER, M.-C. (2011). Conseiller ou accompagner: une pratique qui se cherche. In J.- Y. Robin \& I. Vinatier (Eds.), Conseiller et accompagner. Un défi pour la formation des enseignants, (pp. 139-162). Paris: L'Harmattan.

HENNISSEN, P., CRASBORN, F. BROUWE, N., KORTHAGEN, F., \& BERGEN, T. (2011). Clarifying pre-service teacher perceptions of mentor teachers' developing use of mentoring skills. Teaching and Teacher Education, 27, 1049-1058.

JORRO, A. (2004). Les formateurs et l'accompagnement des professeurs stagiaires dans l'écriture du mémoire professionnel. In A. Gonnin-Bolo \& J.-P. Benoît (Eds.), Le mémoire professionnel en IUFM : Bilan de recherches et questions vives, (pp. 197-207). Saint-Fons: INRP

LAMAURELLE, J.-L. (2010). L'accompagnement professionnel des jeunes enseignants. Hachette éducation, Paris. (livre consulté ONLINE le 27.04.15). 
PAUL, M. (2006). L'accompagmenent : quels enjeux pour le tutorat ? Colloque Tutorat et Accompagnement (pp. 11-24). Bordeaux: AIFRISSS.

RUNTZ-CHRISTAN, E. (2017). Pour comprendre les attentes des stagiaires enseignants à l'égard de leurs enseignants formateurs. Symposium Des interventions éducatives en formation pratique au service du développement de l'identité professionnelle des stagiaires. . CRIFPE.CA.

RUNTZ-CHRISTAN, E.., \& BOUTET, M. (2015). Pour une professionnalisation des enseignants-formateurs : un éclaircissement de la question de l'identité professionnelle est-il nécessaire ? Acte du RIFFEF. Patras.

WITTORSKI, R. (2006). La place du tutorat et de l'accompagnement dans les voies de la professionnalisation. In Colloque "Tutorat et accompagnement », (pp. 25-39). AIFRISSS. [En ligne] Consulté le 09 décembre 2018:

http://www.aifrisss.com/upload/3-colloque-tutorat-et-accompagnement. pdf?PHPSESSID=256d5fa64de1647e6adc7ee8e79e7efd.

EDMÉE RUNTZ - ChRISTAN: Doctorat en études théâtrales et pédagogique de l'Université Paris I Sorbonne. Elle a été collaboratrice à l'Institut pédagogique Recherche et Documentation de Neuchâtel, en Suisse. Elle est actuellement professeur d'enseignement et de recherche à I'Université de Fribourg, en Suisse.

E-mail: edmee.runtz-christan@unifr.ch

Este periódico utiliza a licença Creative Commons Attribution 3.0, para periódicos de acesso aberto (Open Archives Iniciative - OAI). 